\title{
ANÁLISIS DE LA UNIFORMIDAD DE RIEGO EN SISTEMAS DE ASPERSIÓN SEMIPORTÁTIL CON ASPERSORES DE GRAN TAMAÑO
}

\author{
Laserna Arcas, S. ${ }^{1}$, Montero Martínez, J. ${ }^{2}$, Sheikhesmaeili, O. ${ }^{3}$, Alcázar Bascuñana, E. ${ }^{4}$
}

${ }^{1}$ Dr. Ingeniero Agrónomo, Investigador; Centro Regional de Estudios del Agua (CREA), UCLM; Albacete; Santiago.Laserna@uclm.es

${ }^{2}$ Dr. Ingeniero Agrónomo, Titular de Universidad; Centro Regional de Estudios del Agua (CREA), UCLM; Albacete; Jesus.Montero@uclm.es

${ }^{3}$ Ingeniero en Riegos y Drenajes, Investigador; Faculty of Water Sciences Engineering, Shahid Chamran University of Ahvaz, Ahvaz, Iran; omid.sheikh@hotmail.com

${ }^{4}$ Ingeniera Agrónomo, Investigadora; Escuela Técnica Superior de Ingenieros Agrónomos y de Montes, UCLM; Albacete; elena.alcazar@outlook.com

\section{Resumen}

La sostenibilidad en la agricultura de regadío depende en gran medida de conseguir una alta eficiencia de aplicación en el riego. Es muy importante conocer los factores que afectan a la uniformidad de riego, especialmente en aspersores semi-portátiles de gran tamaño, que son un sistema muy común en áreas áridas y semiáridas, como Irán. Hasta el momento, la uniformidad de distribución del agua aplicada no ha sido considerada cuantitativamente en la mayoría de las combinaciones de variables hidráulicas y meteorológicas con aspersores portátiles de gran tamaño. En este trabajo, se ha caracterizado el coeficiente de uniformidad (CU) analizando la influencia de los principales factores que le afectan, como la velocidad del viento $(W)$, la presión de trabajo $(P)$ y el marco de riego. Los ensayos de campo se realizaron con un solo aspersor al aire libre. Se aprecia un efecto significativo del viento, como parámetro meteorológico, sobre el CU bajo diferentes condiciones climáticas, en relación a la presión y la separación entre aspersores. Este comportamiento es muy similar al obtenido con aspersores de tamaño medio. Los criterios técnicos propuestos en los resultados se pueden utilizar para optimizar la gestión del riego por aspersión de acuerdo con factores de diseño adecuados para una amplia gama de condiciones climáticas y presión (es decir 450 y $500 \mathrm{kPa}$ ). Así, la relación entre la separación entre aspersores y el radio mojado no debe superar los 0,45 con el fin de alcanzar el coeficiente de diseño de uniformidad aceptable ( $80 \%)$ bajo condiciones de viento $\left(>2 \mathrm{~m} \mathrm{~s}^{-1}\right)$ en el sistema de riego.

\section{1.- Introducción. Objetivos}

La sostenibilidad en la agricultura de regadío bajo sistemas de riego a presión aplicado a áreas con mucho viento y semiáridas depende en gran medida de lograr una alta eficiencia de riego mediante un adecuado diseño y manejo del sistema. Muchos trabajos se han llevado a cabo para analizar el papel de los parámetros que influyen en la eficiencia y uniformidad de distribución del agua en sistemas de riego por aspersión, pero sobre todo, con aspersores de tamaño medio. Sin embargo, la bibliografía disponible no aborda los problemas de mejorar el coeficiente de uniformidad (CU) influenciado por la combinación de variables hidráulicas y meteorológicas con aspersores semi-portátiles de gran tamaño. No 
hay estudios realizados con aspersores de gran tamaño que trabajen con altos caudales (mayor de $3 / \mathrm{s} \mathrm{s}^{-1}$ ) y gran separación entre aspersores (superior a $24 \mathrm{~m}$ ).

La aspersión semi-portátil con aspersores de gran tamaño es un sistema de riego muy popular y extendido en zonas de regadío con condiciones climáticas áridas y semiáridas. En Irán, el $48 \%$ de la superficie total de riego a presión o el $85 \%$ de la superficie de riego por aspersión está cubierta por este sistema (660.000 ha) (Report, 2014).

La uniformidad en la distribución del agua es un indicador de las variaciones espaciales aplicadas sobre la superficie de regadío. Este factor de diseño afecta a aspectos importantes de la agricultura como, la eficiencia del uso del agua, la lixiviación de fertilizantes y el rendimiento del cultivo (Seginer et al., 1991a). Está demostrado que un aumento de la uniformidad en la aplicación del agua en el campo puede favorecer la eficiencia del riego mediante la reducción de la percolación profunda y la escorrentía superficial causada por un exceso de riego. Por lo tanto, las evaluaciones de campo son un excelente procedimiento para investigar los factores que afectan a la uniformidad del riego real bajo múltiples combinaciones de condiciones climáticas e hidráulicas en los sistemas de riego por aspersión. Por otra parte, una distribución no uniforme no sólo podría dejar algunas parte de la cosecha en una situación deficitaria de agua, sino que también podría exceder el riego de otras partes causando acumulaciones de agua, daños a las plantas, la salinización del suelo, y la lixiviación de fertilizantes químicos en las aguas subterráneas (Solomon, 1983).

Las variables meteorológicas, como la velocidad y dirección del viento $(\mathrm{W})$ son los principales parámetros que tienen mayor impacto en el modelo de distribución de agua de riego por aspersión, jugando un papel importante en el arrastre del viento y las perdidas por evaporación (Dechmi et al., 2003; Tarjuelo et al., 2000; Keller y Bliesner, 1990). Estas referencias han dado lugar a dos importantes conclusiones: en primer lugar, el modelo de distribución de agua de un aspersor aislado se distorsiona y reduce bajo condiciones de viento; en segundo lugar, el agua aplicada podría perderse parcialmente por evaporación, especialmente por el arrastre fuera de la superficie de riego. La consecuencia de estos problemas podrían ser el riego excesivo o insuficiente riego de partes del campo.

Muchos autores indican que, aunque la velocidad del viento es el parámetro meteorológico más importante que afecta a los indicadores del uso del agua en el campo de los sistemas de riego por aspersión (Tarjuelo et al., 1999a y b; Sánchez et al., 2010), su influencia depende enormemente de los parámetros del diseño del sistema, tales como la presión de los aspersores y su separación, el tamaño de la boquilla o el tipo de aspersor (Keller y Bliesner, 1990).

Burt et al. (1997) indicaron que los factores más influyentes en la heterogeneidad de la distribución del agua son las variaciones de presión, el diseño del aspersor, el marco de riego, y sobre todo las condiciones climáticas, tales como la velocidad del viento.

Según Keller y Bliesner (1990), la mayoría de los sistemas de riego por aspersión requieren un valor mínimo de Coeficiente de Uniformidad de Christiansen (CU) $\geq 80 \%$. Bralts et al (1994) indicaron que un aumento del $5-12 \%$ en el CU podría supones el 3-17\% más de rendimiento en el grano de trigo. Los bajos valores de CU indican generalmente una combinación defectuosa del número y tamaño de boquillas, presión y separación entre aspersores (Tarjuelo et al., 1999a).

Otro de los factores decisivos del sistema para mejorar la uniformidad de distribución de agua es el grado de solapamiento de los aspersores. Los sistemas de riego por aspersión requieren un solapamiento adecuado entre aspersores para obtener una buena uniformidad de distribución (Keller y Bliesner, 1990). Phocaides (2007) recomienda que, con el fin de obtener una buena uniformidad por solapamiento de aspersores, esta no debería exceder el $65 \%$ del diámetro mojado en condiciones de viento bajas a moderadas, para marcos cuadrados y rectangulares. También afirmó que, en condiciones de viento fuertes, la separación entre aspersores debería ser del 50\% del diámetro mojado con la dirección lateral perpendicular a la dirección del viento. 
Los objetivos de este documento son: (1) caracterizar el CU bajo diferentes escenarios de funcionamiento de presión para una amplia gama de condiciones climáticas en sistema de riego con aspersores semi-portátiles de gran formato; (2) analizar la uniformidad de riego en respuesta a los diversos escenarios evaluados de la combinación de los parámetros climáticos influyentes como la velocidad del viento y las características del diseño del sistema de aspersión incluyendo la presión, la separación y el marco de riego, y (3) dar algunas recomendaciones para ayudar en el diseño de proyectos y la gestión práctica en sistemas de riego por aspersión con aspersores de gran tamaño semi-portátiles en zonas semiáridas.

\section{2.- Material y métodos}

\section{1.- Localización de los ensayos}

Los ensayos al aire libre se realizaron en la zona sur-oeste de Irán (Región

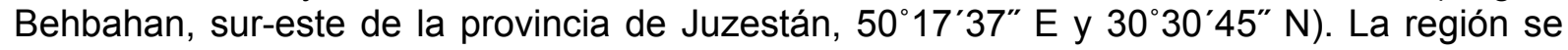
caracteriza por un clima semiárido, con inviernos suaves y veranos calurosos. La evotranspiración anual y la precipitación media son de 1717 y $350 \mathrm{~mm}$, respectivamente. El sistema de riego por aspersión típico en este área son los aspersores semi-portátiles de gran tamaño (Sheikhesmaeili, 2003). Actualmente, este sistema de riego es la configuración principal en ciertas regiones iraníes, como la provincia de Juzestán, donde está cubierto por el 73,1\% (22.634 ha) de la superficie total de riego a presión o $99 \%$ de la superficie de riego por aspersión (Report, 2014). En este sistema de aspersores de gran tamaño portátil, toda la red de tuberías está enterrada y sólo los aspersores se mueven manualmente sobre el lateral, que se puede extender hasta $300 \mathrm{~m}$.

\section{2.- Ensayos de un aspersor al aire libre}

Para analizar la uniformidad en sistemas de riego con aspersores de gran tamaño, se realizaron cuarenta ensayos de un aspersor aislado al aire libre, siguiendo las normas ISO 15886-3 (2012) y ASAE S398.1 (2001). El tipo de aspersor de impacto de gran tamaño utilizado en estas pruebas fue el A-D-5 (Abyaran Dasht Inc., Tehran, Iran). Este aspersor tiene tres boquillas $(11+6,2+3,2 \mathrm{~mm})$ y es similar al aspersor VYR 155 (VIRSA Inc., Burgos, España) (figura 1). Los dos escenarios de presiones $(P)$ de trabajo utilizados fueron las recomendadas por el fabricante (450 y $500 \mathrm{kPa}$ ), con un caudal descargado de 3,25 y 3,46 $\mathrm{I} \cdot \mathrm{s}^{-1}$, respectivamente. Las pruebas se realizaron en los meses de abril a noviembre durante diferentes horas del día para cubrir todas las condiciones climáticas. En estas pruebas se utilizó una red de pluviómetros de 22 × 22 filas y columnas de pluviómetros (figura 2). Las boquillas de los aspersores se colocaron $1,35 \mathrm{~m}$ por encima de la abertura del pluviómetro. Los pluviómetros, con un diámetro de abertura de $0,1 \mathrm{~m}$ y $0,15 \mathrm{~m}$ de altura, se colocan en una cuadrícula de $3 \times 3 \mathrm{~m}^{2}$. El agua procede de un sondeo; una válvula regula la presión del agua, y se utilizó un caudalímetro en línea calibrado (con un rango de error $<2 \%$ ) para medir la cantidad total de agua bombeada. La presión de trabajo en el aspersor se midió utilizando un medidor de presión de tubo Pitot en el centro del chorro del aspersor, aproximadamente a $1,5 \mathrm{~mm}$ de la boquilla. Las condiciones climáticas (la temperatura del aire, la humedad relativa y la velocidad y dirección del viento a 2 metros de altura) se midieron en una estación meteorológica automática situada a $50 \mathrm{~m}$ del lugar de ensayo a intervalos de 5 minutos durante la duración de la prueba. La tabla 1 resume los valores medios, así como los máximos y mínimos, de los parámetros ambientales (temperatura, humedad y velocidad del viento) durante los ensayos.

La duración de las pruebas fue de una hora. Una vez finalizado el ensayo, el volumen de agua recogida en los pluviómetros se midió con una probeta calibrada, con la particularidad de seguir siempre el mismo orden de lectura. En cada pluviómetro, el volumen 
de agua recogido se corrigió para cuantificar las pérdidas por evaporación durante el tiempo de riego (una hora).

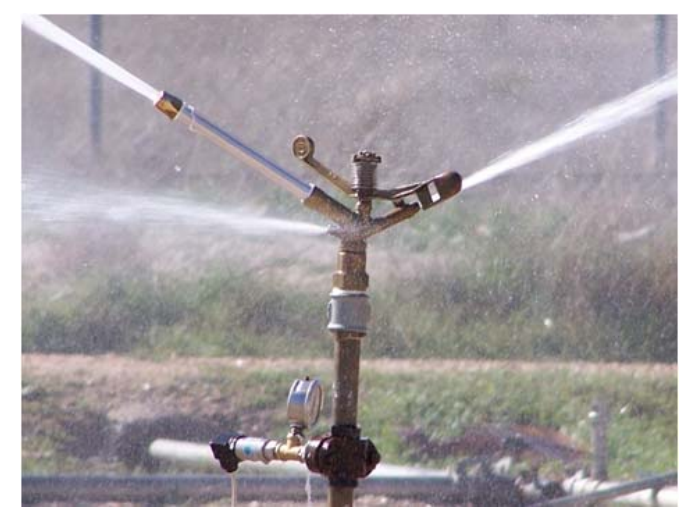

Figura 1. Detalle del aspersor de gran tamaño A-D-5 con tres boquillas
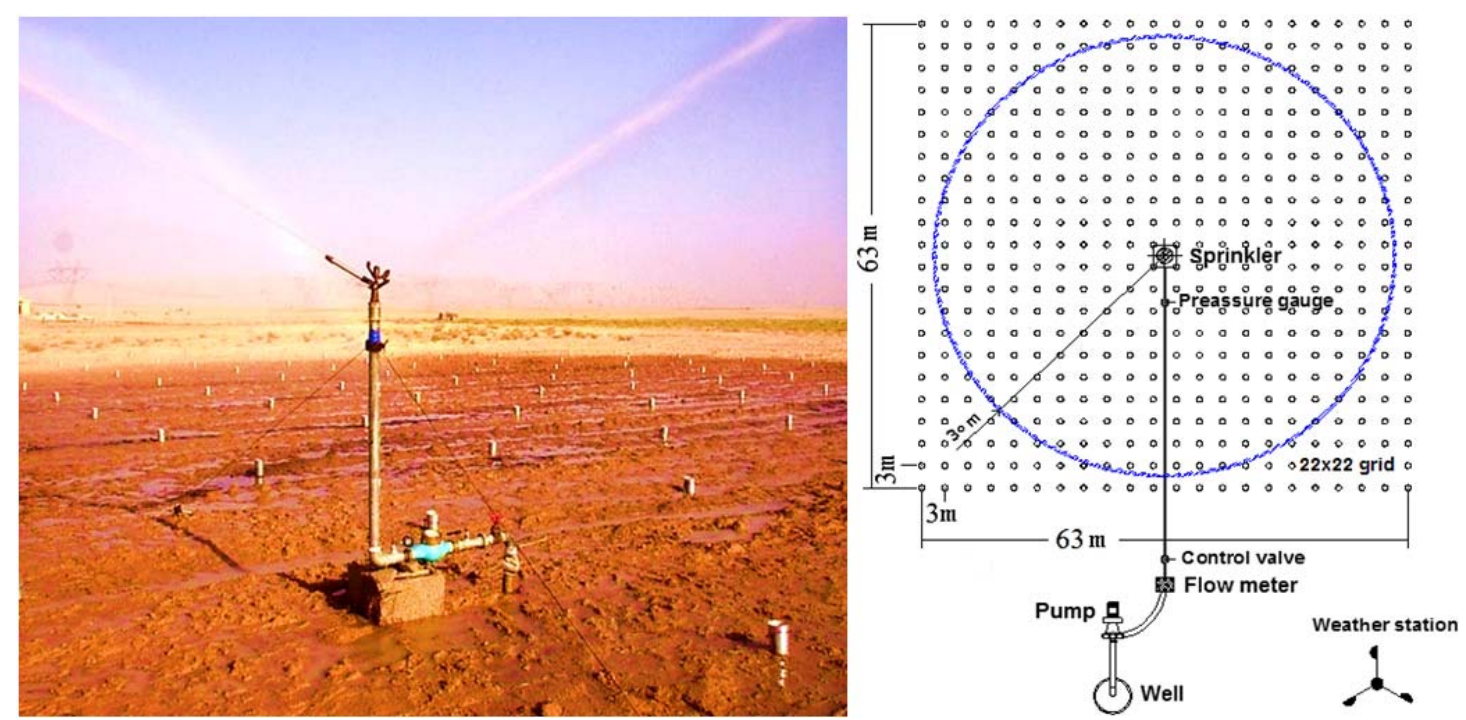

Figura 2. Esquema del ensayo del aspersor aislado al aire libre

Tabla 1. Media de los valores medidos de los parámetros meteorológicos (y valores mínimo y máximo) para los 40 ensayos experimentales

\begin{tabular}{ccc}
\hline $\mathbf{W}(\mathbf{m} / \mathbf{s})$ & $\mathbf{T}\left({ }^{\circ} \mathbf{C}\right)$ & $\mathbf{H ~ ( \% )}$ \\
\hline $1.62(0-6.8)$ & $31.9(21.4-44.9)$ & $41.8(11.8-80.0)$ \\
\hline
\end{tabular}

\section{3.- Caracterización del patrón de distribución de agua por aspersión sin viento}

Con el fin de caracterizar el patrón de distribución de agua del aspersor A-D-5 se realizaron varios ensayos, de conformidad con la norma ISO 15886-3 (2012), bajo condiciones sin viento en las instalaciones del Centro Regional de Estudios del Agua (CREA) de la Universidad de Castilla-La Mancha (Albacete, España). Los ensayos se realizaron con el aspersor con tres boquillas $(11+6,2+3,2 \mathrm{~mm})$, a dos presión de trabajo, 450 y $500 \mathrm{kPa}$. El aspersor se dispuso a 1,5 metros sobre el suelo. Se colocaron dos líneas de 
pluviómetros de 0,16 $\mathrm{m}$ de diámetro formando un ángulo de $90^{\circ}$ con una separación de $1 \mathrm{~m}$ entre ellos. Se hicieron dos repeticiones de cada presión de trabajo. El volumen de agua de cada uno de los pluviómetros se determinó como el promedio de las dos filas de pluviómetros y para ambas repeticiones.

\section{4.- Medida del tamaño de gota de los aspersores}

Con el fin de establecer la relación entre el CU y el tamaño de gota del aspersor, se utilizó un disdrómetro óptico modelo ODM 470, fabricado por Eigenbrodt (Königsmoor Inc., Alemania), a las presiones de trabajo de 450 y $500 \mathrm{kPa}$. Se puede encontrar una descripción detallada del equipo en Montero et al. (2006). El dispositivo se basa en la atenuación de un haz de luz infrarroja cuando las gotas cruzan a través de una ventana óptica. La forma del detector del haz es circular con un diámetro de $20 \mathrm{~mm}$. El disdrómetro realiza mediciones continuas de las gotas emitidas por el aspersor. Cada gota produce una atenuación en el haz luminoso. El análisis de la señal permite estimar el diámetro de la gota y el tiempo que tarda en atravesar el haz de luz (tiempo de paso).

La medida del tamaño de gota se realizó al mismo tiempo que las pruebas radiales. Las distancias al aspersor fueron de 4, 8, 12, 16, 20, y $24 \mathrm{~m}$. Para cada distancia, se calculó el Diámetro Mediano de Volumen (VMD), que es el diámetro de gota al que le corresponde el $50 \%$ del volumen de agua acumulada (Montero et al., 2003). La medida de la distribución de las gotas engloba las gotas arrojadas por el conjunto de las tres boquillas. La trayectoria de los ángulos para cada boquilla es $28^{\circ}-28^{\circ}-13^{\circ}$.

\section{5.- Análisis del Coeficiente de Uniformidad de Christiansen (CU)}

La distribución de agua medida para un aspersor aislado bajo diferentes condiciones de viento, se solapó para determinar la uniformidad de riego en dos tipos de marcos de aspersores (cuadrado y rectangular), con tres separaciones entre aspersores $(21,24$ y 30 $\mathrm{m})$

La disposición de los marcos, Se (separación entre aspersores dentro de cada línea, en $\mathrm{m}$ ) $\mathrm{x}$ SI (separación entre líneas, en $\mathrm{m}$ ), fue la siguiente: $21 \times 21,24 \times 24$ y $30 \times 30$ para marcos cuadrados, y $21 \times 24,21 \times 30$ y $24 \times 30$ para marcos rectangulares.

El parámetro calculado para determinar la uniformidad de riego fue el Coeficiente de Uniformidad de Christiansen (Christiansen, 1942).

Se realizó un análisis de varianza (ANOVA) y la prueba Tukey HSD, para determinar los efectos significativos de las variables analizadas ( $P, W, S I$ y Se) sobre el $\mathrm{CU}$, utilizando los software SPSS v.18 (SPSS Inc.) y EXCEL 2010 (MicroSoft Inc.).

\section{3.- Resultados y discusión}

\section{1.- Curvas radiales y distribución de los tamaños de gota}

Una representación gráfica de las curvas pluviométricas del aspersor obtenidas en los ensayos radiales se muestra en la figura 3a. En ella, se puede observar unas curvas con forma triangular. También se observa que el volumen de aplicación es más alto cerca del aspersor $(8-10 \mathrm{~cm})$, y luego disminuye hasta el final. Esto es indicativo del buen comportamiento previsible del aspersor en condiciones de poco viento, pero no tan bueno cuando haga fuertes vientos (Tarjuelo et al, 1999a). Respecto a las medidas de los tamaños de gota para el aspersor, se calculó la VMD para cada distancia, representándolos gráficamente en la figura $3 b$.

No se observaron diferencias significativas en la distribución del tamaño de las gotas entre las dos presiones (450 y $500 \mathrm{kPa}$ ). En relación con las distancias al aspersor, cuando 
mayor es la distancia, mayores son los tamaños de gota, siguiendo una tendencia exponencial. Para estos aspersores, con diámetros de boquilla grande y altas presiones (450-500 kPa), se forman un rango de tamaño de gota similares (hasta 3,5-4 mm) a los aspersores de tamaño medio, con boquillas pequeñas y presiones medias (250-350 kPa) (Li et al., 1994; Montero et al., 2003 y 2006).

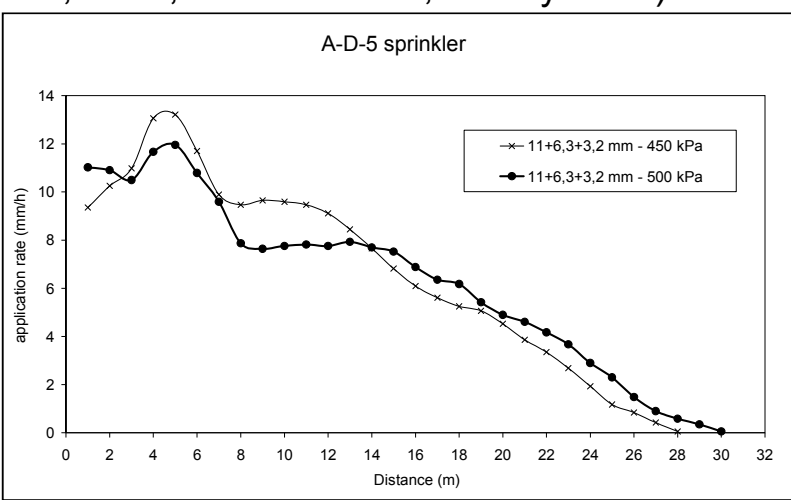

(a)

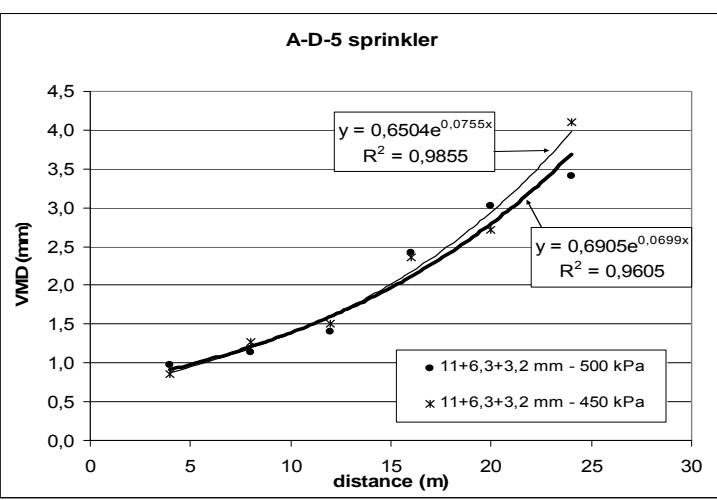

(b)

Figura 3. Distribución radial (a) y distribución de los tamaños de gota (b), para las presiones de 450 y $500 \mathrm{kPa}$

\section{2.- Análisis del Coeficiente de Uniformidad al aire libre}

Los patrones de distribución del agua obtenidos bajo diversas condiciones de viento y presiones de trabajo, se solaparon para calcular los valores de CU para los diferentes marcos de riego definidos. La tabla 2 resume los valores resultantes de $\mathrm{CU}$ de todos los escenarios simulados. Los datos se clasifican en tres intervalos de viento diferentes (bajo: $\mathrm{W}<2 \mathrm{~m} \mathrm{~s}^{-1}$, moderado: $2-4 \mathrm{~m} \mathrm{~s}^{-1} \mathrm{y}$ alto: $\mathrm{W}>4 \mathrm{~m} \mathrm{~s}^{-1}$ ).

Tabla 2 Valores de CU obtenidos en los diferentes ensayos

\begin{tabular}{|c|c|c|c|c|c|c|c|c|c|}
\hline \multirow{3}{*}{$\begin{array}{c}W \\
\left(m^{-1}\right)\end{array}$} & \multirow{3}{*}{$\begin{array}{c}\text { Presión } \\
\text { (kPa) }\end{array}$} & \multicolumn{6}{|c|}{ Marco de riego $(\mathrm{mxm})$} & \multirow{2}{*}{\multicolumn{2}{|c|}{ Valores Medios }} \\
\hline & & \multicolumn{3}{|c|}{ Cuadrado } & \multicolumn{3}{|c|}{ Rectangular } & & \\
\hline & & $21 \times 21$ & $24 \times 24$ & $30 \times 30$ & $21 \times 24$ & $21 \times 30$ & $24 \times 30$ & $\begin{array}{l}\text { Por } \\
\text { presión }\end{array}$ & Global \\
\hline \multirow{2}{*}{$<0$} & 450 & 90,5 & 83,3 & 79,7 & 87,0 & 86,9 & 83,7 & 85,2 & \multirow{2}{*}{86,1} \\
\hline & 500 & 91,7 & 86,1 & 83,2 & 87,5 & 87,6 & 86,1 & 87,0 & \\
\hline \multirow{2}{*}{$2-4$} & 450 & 76,0 & 74,7 & 66,4 & 74,0 & 67,0 & 68,4 & 71,1 & \multirow{2}{*}{74,8} \\
\hline & 500 & 79,0 & 77,5 & 76,2 & 79,2 & 80,5 & 78,9 & 78,6 & \\
\hline \multirow{2}{*}{$>4$} & 450 & 78,2 & 78,3 & 61,5 & 76,9 & 66,6 & 68,4 & 71,6 & \multirow{2}{*}{68,4} \\
\hline & 500 & 72,4 & 70,0 & 59,0 & 69,9 & 59,6 & 60,3 & 65,2 & \\
\hline \multirow{3}{*}{ Media } & 450 & 85,1 & 80,6 & 73,1 & 82,3 & 78,7 & 77,5 & 79,6 & \multirow{2}{*}{80,2} \\
\hline & 500 & 85,9 & 81,6 & 77,5 & 82,8 & 81,2 & 80,1 & 81,5 & \\
\hline & Global & $\begin{array}{l}85,3 \\
(\mathrm{~A})\end{array}$ & $\begin{array}{c}81,0 \\
(B)\end{array}$ & $\begin{array}{c}74,6 \\
(\mathrm{C})\end{array}$ & $\begin{array}{l}82,5 \\
(A B)\end{array}$ & $\begin{array}{l}79,5 \\
(\mathrm{~B})\end{array}$ & $\begin{array}{l}78,3 \\
(\mathrm{BC})\end{array}$ & & \\
\hline
\end{tabular}

$\left.{ }^{*}\right)$ Los paréntesis muestras grupos homogéneos obtenidos después de realizar el análisis de varianza (grupo de filas)

Efectos de la presión de trabajo sobre el Coeficiente de Uniformidad:

Como el tamaño de gota está controlado por la presión de funcionamiento y las boquillas del aspersor, es muy importante que el aspersor funcione en el rango adecuado de 
presiones (es decir, 450 a $500 \mathrm{kPa}$ ) recomendado por el fabricante. Se realizó la prueba estadística ANOVA para comprobar el efecto de la presión en el CU cuando se cambia de $450 \mathrm{kPa}$ a $500 \mathrm{kPa}$. Los resultados estadísticos muestran que no hay diferencia significativa entre los valores de CU sin diferenciación de la velocidad del viento, debido a la similitud de los modelos de distribución radial en las dos presiones $(p=0,13,>0,05)$. Así, y de acuerdo con Keller y Bliesner (1990), se recomienda evitar trabajar con presiones superiores a 450 $\mathrm{kPa}$, ya que una mayor presión implica un mayor coste económico energético para obtener el mismo valor de CU.

Sin embargo, cuando la influencia de la presión se analiza con la diferenciación en clases de velocidad de viento, se observa como la presión produce diferencias significativas en CU. De acuerdo con la tabla 2, los valores de CU para baja y media W son altos en 500 $\mathrm{kPa}(87,7 \%-78,6 \%)$ en comparación con los $450 \mathrm{kPa}(85,9 \%-71,1 \%)$, pero cuando se incrementa W por encima de $4 \mathrm{~m} \cdot \mathrm{s}^{-1}$, influye negativamente este efecto sobre el CU para $\mathrm{P}=500 \mathrm{kPa}(\mathrm{CU}=65,9 \%)$ en comparación con $450 \mathrm{kPa}(\mathrm{CU}=73,0 \%)$.

Estos resultados concuerdan con las conclusiones de Li (1997), que demostró que la formación de los tamaños de gota estaba influenciado principalmente por la presión, y es una razón importante para explicar que las gotas pequeñas resultantes de una alta presión pueden perturbar gravemente el modelo de aplicación del agua y ser también el motivo de las pérdidas por el viento y la evaporación.

\section{Efectos de la velocidad del viento en el coeficiente de uniformidad}

De acuerdo con los análisis de varianza mencionados realizados, se encontraron diferencias significativas en la variación de la uniformidad debidas a la velocidad del viento $\left(p=3,0 \cdot 10^{-9},<0,05\right)$, de modo que el $\mathrm{CU}$ disminuye con el aumento de $\mathrm{W}$, aunque no linealmente. La figura 4 muestra la variación de los valores de CU frente a W para los diferentes marcos de riego y a la presión de trabajo de $450 \mathrm{kPa}$.

De acuerdo con Tarjuelo et al. (1999a) el mejor coeficiente de determinación $\left(R^{2}\right)$ se consiguió gracias a las ecuaciones polinómicas de tercer grado, debido a que $\mathrm{W}$ tuvo un efecto negativo en los valores del CU por encima de una velocidad mínima del viento $(\approx 2$ $\left.\mathrm{m} \cdot \mathrm{s}^{-1}\right)$, mientras que con vientos fuertes $\left(\mathrm{W}>4 \mathrm{~m} \cdot \mathrm{s}^{-1}\right)$ los valores de CU se mantuvieron casi constantes.
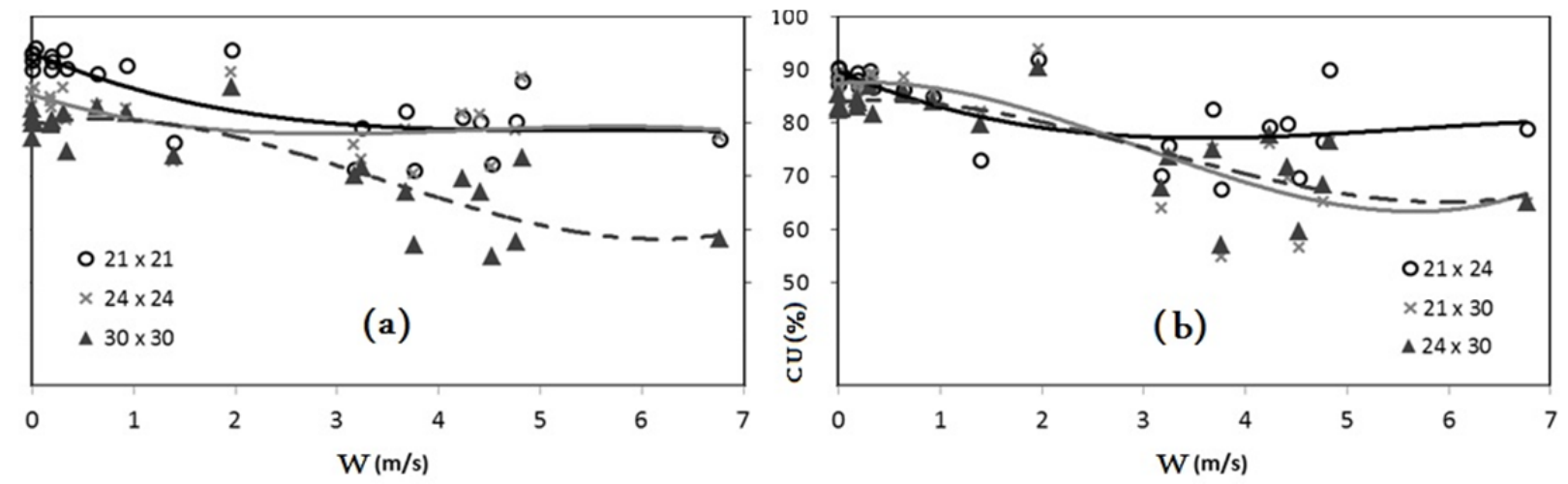

Figura 4. Coeficiente de uniformidad vs velocidad del viento para diferentes marcos de riego, en cuadrado (a) y en rectángulo (b), a $450 \mathrm{kPa}$

Los resultados indican que los valores de $\mathrm{CU}$ aumentan ligeramente en condiciones de baja velocidad del viento $\left(\mathrm{W}<2 \mathrm{~m} \cdot \mathrm{s}^{-1}\right)$ conforme se pasa de 450 a $500 \mathrm{kPa}$, según la tabla 2. Se confirman los resultados de De Lima y Torfs (1994), quienes explicaron que el mayor efecto del $\mathrm{W}$ afecta al tener gotas más pequeñas, debido principalmente a una mayor fricción y menor velocidad de caída, estando más sometidas a la acción del viento. Por otro lado, el CU cae por debajo del $75 \%$ cuando $W$ es superior a $3,5-4 \mathrm{~m} \cdot \mathrm{s}^{-1}$, que según Phocaides (2007), es la velocidad crítica que recomienda para la aplicación de riego por aspersión. De acuerdo con Yacoubi et al. (2012), se puede afirmar que la velocidad del 
viento superior a $4 \mathrm{~m} \cdot \mathrm{s}^{-1}$ afecta significativamente al CU (76,3\%), independientemente de las separaciones entre aspersores y la presión.

La tabla 2 confirma las conclusiones de Seginer et al. (1991b) que indica que la distribución del agua se distorsiona en gran medida para una velocidad del viento mayor a 4 $\mathrm{m} \cdot \mathrm{s}^{-1}$, especialmente cuando la presión aumenta a $500 \mathrm{kPa}$, ya que el efecto anteriormente mencionado de la distorsión del modelo de gotas, no es tan evidente a $450 \mathrm{kPa}$ de presión.

\section{Efectos de la separación entre aspersores y el marco en el coeficiente de uniformidad}

El resultado del test ANOVA mostró que no existen diferencias significativas entre los valores del CU en los marcos cuadrados y rectangulares analizados $(p=0,7911,>0,05)$. Por lo tanto, no es posible concluir qué tipo de marco es más adecuado, cuadrado o rectangular, para el sistema de aspersión analizado y para esta configuración de boquillas. Como se demuestra a continuación, dependerá de la separación entre aspersores seleccionada.

Los resultados muestran claramente como la separación entre aspersores (Se y SI) sí tienen influencias significativas sobre el CU $\left(p=5,75 \cdot 10^{-5},<0,05\right)$. Se puede confirmar la conclusión de Montero et al. (2004), quienes afirmaron que el parámetro de diseño más importante es la separación entre aspersores, después de analizar la influencia de parámetros de diseño y rendimiento, tales como la disposición de la subunidad, la separación entre laterales, la presión, la pluviometría del sistema y la eficiencia de aplicación en el coste de la aplicación del agua, en un sistema de riego por aspersión fijo.

El test de Tukey HSD se ha aplicado con el objetivo de revelar las diferencias significativas del Se y SI en el CU. La tabla 3 muestra los grupos homogéneos para cada marco de riego, diferenciando en las pruebas globales, la presión de trabajo, o las velocidades del viento. De acuerdo con los resultados mostrados en la tabla 3 , los valores medios de CU disminuyen mientras Se y SI aumentan, obteniendo tres grupos homogéneos y destacando que los marcos $21 \times 24,24 \times 24,21 \times 30$ y $24 \times 30$ se incluyen en el mismo grupo, por lo que no hay diferencias significativas entre los valores del CU obtenidos para estos marcos, incluso diferenciando la presión de trabajo.

Tabla 3. Grupos homogéneos de CU para los diferentes marcos de riego, diferenciando por presiones de trabajo y velocidad del viento.

\begin{tabular}{|c|c|c|c|c|c|c|c|c|c|c|c|}
\hline \multirow{3}{*}{$\begin{array}{c}\text { MARCO } \\
\text { Tipo } \\
30 \times 30\end{array}$} & \multirow{2}{*}{\multicolumn{2}{|c|}{$\begin{array}{l}\text { Global } \\
\text { Todas P y W } \\
\text { Media }\end{array}$}} & \multicolumn{4}{|c|}{ Por presión (P) } & \multicolumn{5}{|c|}{ Por velocidad del viento (W) } \\
\hline & & & \multicolumn{2}{|c|}{$\begin{array}{l}450 \mathrm{kPa} \\
\text { Media }\end{array}$} & \multicolumn{2}{|c|}{$\begin{array}{l}500 \mathrm{kPa} \\
\text { Media }\end{array}$} & \multicolumn{2}{|c|}{$\begin{array}{c}<2 \mathrm{~m} \mathrm{~s}^{-1} \\
\text { Media }\end{array}$} & $\begin{array}{r}2-4 \mathrm{~m} \mathrm{~s}^{-1} \\
\text { Media }\end{array}$ & \multicolumn{2}{|c|}{$\begin{array}{r}>4 \mathrm{~m} \mathrm{~s}^{-1} \\
\text { Media }\end{array}$} \\
\hline & 74,6 & $\mathrm{C}$ & 73,1 & $C$ & 77,5 & $\mathrm{~B}$ & 80,9 & $\mathrm{D}$ & $69,7 \quad A$ & 60,8 & B \\
\hline $24 \times 30$ & 78,3 & $B C$ & 77,5 & $\mathrm{BC}$ & 80,1 & $\mathrm{~B}$ & 84,5 & C & 71,9 A & 66,1 & B \\
\hline $21 \times 30$ & 79,5 & B & 78,7 & B & 81,2 & $\mathrm{BC}$ & 87,2 & B & $71,5 \quad A$ & 64,6 & B \\
\hline $24 \times 24$ & 81,0 & B & 80,6 & $A B$ & 81,6 & $\mathrm{BC}$ & 84,3 & C & $75,1 \quad A$ & 75,9 & $A$ \\
\hline $21 \times 24$ & 82,5 & $A B$ & 82,3 & $A B$ & 82,8 & $\mathrm{BC}$ & 87,2 & B & $75,8 \quad A$ & 74,9 & $A$ \\
\hline $21 \times 21$ & 85,3 & $A$ & 85,1 & $A$ & 85,9 & C & 90,9 & $A$ & $77,0 \quad \mathrm{~A}$ & 76,5 & $A$ \\
\hline
\end{tabular}

Centrándonos en el análisis de CU por intervalos de $W$, para bajo viento los resultados muestran (tabla 3 ) que un aumento de $24 \mathrm{~m}$ hasta $30 \mathrm{~m}$ en la separación entre líneas (SI) no tiene diferencias significativas en el valor de CU cuando "Se" se encuentra a $21 \mathrm{~m}$ (grupo B) o $24 \mathrm{~m}$ (grupo C). Por lo tanto, es importante tenerlo en cuenta en el diseño del sistema de riego puesto que un aumento en la separación entre líneas SI supone soluciones más económicas, incluso, en este sistema semi-portátil en particular, pues implica reducir el tiempo requerido para mover todos los aspersores y, en consecuencia, disminuyen los tiempos muertos entre riegos. 
Sin embargo, seleccionar una separación máxima de aspersores de hasta $30 \mathrm{~m}$ podría originar una disminución importante en el valor de CU cuando aumenta la velocidad del viento por encima de $4 \mathrm{~m} \cdot \mathrm{s}^{-1}$, obteniendo valores del CU $10-15 \%$ más bajos que con separaciones entre aspersores de $21 \mathrm{~m}$ y $24 \mathrm{~m}$. De esta manera, un análisis minucioso de las tablas 2 y 3 revela que todos los marcos cumplen el CU aceptable (80\%) bajo condiciones de poco viento, pero cuando el viento supera este valor, disminuye el CU por debajo del $80 \%$, obteniendo $\mathrm{CU} \approx 75 \%$ para $\mathrm{Se}<30 \mathrm{~m}$.

Por lo tanto, se llega a la conclusión de que una buena solución técnico-económica sería un marco de riego cuadrado de $24 \times 24$ o rectangular de 21 × 24 a $450 \mathrm{kPa}$, dependiendo del viento predominante para la superficie de riego. Hoy en día, la separación entre aspersores "Se" que se utiliza con mayor frecuencia en el sistema de riego por aspersión semi-portátil en Irán es de $25 \times 30$ (Sheikhesmaeili, 2003), para las condiciones recomendadas en este estudio (presiones hasta $450 \mathrm{kPa}$ y $\mathrm{W}<4 \mathrm{~m} \cdot \mathrm{s}^{-1}$. Para facilitar la ejecución y el mantenimiento es recomendable seleccionar "Se" múltiplo de $5 \mathrm{~m}$, como $25 \times 30$ en lugar de $24 \times 30$. Por otro lado, una buena recomendación del Se=21 m (o $20 \mathrm{~m}$ ) es que puede ser aplicado en vientos por encima de $4 \mathrm{~m} \cdot \mathrm{s}^{-1}$ con un CU aceptable $(79,4 \%$, según la tabla 2).

Por último, y como resumen de los resultados, la tabla 4 se podría utilizar para seleccionar la mejor separación entre aspersores en el sistema de riego semiportátil con aspersores de gran formato.

Tabla 4. Recomendación de la separación entre aspersores (Se $x$ SI) bajo diferentes velocidades de viento, presión de trabajo y condiciones de solapamiento.

\begin{tabular}{cccccc}
\hline $\begin{array}{c}\text { Viento } \\
\left(\mathbf{m ~ s}^{-1}\right)\end{array}$ & $\begin{array}{c}\text { Se } \times \mathbf{S I} \\
(\mathbf{m} \times \mathbf{m})\end{array}$ & $\begin{array}{c}\mathbf{P} \\
(\mathbf{k P a})\end{array}$ & $\begin{array}{c}\text { Diámetro } \\
\mathbf{m o j a d o} \\
\text { Dw }(\mathbf{m})\end{array}$ & Se/Dw & SI/Dw \\
\hline \hline 2 & $30 \times 30$ & 500 & 60 & 0,50 & 0,50 \\
& $25 \times 30$ & 450 & 56 & 0,45 & 0,54 \\
$2-4$ & $25 \times 25$ & 450 & 56 & 0,45 & 0,45 \\
$>4$ & $25 \times 25$ & 450 & 56 & 0,45 & 0,45 \\
\hline
\end{tabular}

\section{4.- Conclusiones}

La distribución de agua en sistemas de riego por aspersión con aspersores de gran tamaño es muy similar a la producida con aspersores de tamaño medio. Los resultados de este estudio sugieren que es mejor trabajar con una presión de trabajo de $450 \mathrm{kPa}$ frente a $500 \mathrm{kPa}$, a pesar de utilizar boquillas con un gran diámetro. La influencia del viento es significativa sobre el patrón de distribución de agua, sobre todo para velocidades mayores de $2 \mathrm{~m} / \mathrm{s}$. Coeficientes de uniformidad aceptables se pueden conseguir incluso con marcos de riego de $24 \times 30 \mathrm{~m}^{2}$ y $30 \times 30 \mathrm{~m}^{2}$ en situaciones de riegos nocturnos con bajo viento, a la presión de $450 \mathrm{kPa}$. Como consecuencia de este estudio, unos criterios técnicos pueden ser usados para optimizar el manejo del riego con aspersores de gran tamaño, para diferentes escenarios de trabajo que incluyen el marco de riego, la presión de trabajo y el efecto del viento.

\section{5.- Bibliografía}

ASAE. (2001). Standard S398.1: Procedure for Sprinkler Testing and Performance Reporting. ASAE Standards. ASABE, St. Joseph, MI. USA.

Bralts, VF, Pandey, SR \& Miller, A. (1994). Energy saving and irrigation performance of a modified center pivot irrigation systems. App Eng Agric 10(1): 27-36. 
Burt, CM, Clemmens, AJ, Strelkoff, TS, Solomon, KH, Bliesner, RD, Hardy, LA, Howell, TA \& Eisenhauer, DE. (1997). Irrigation Performance Measures: Efficiency and Uniformity. J Irrig Drain Eng 123(6): 423-442.

Christiansen, JE. (1942). Irrigation by sprinkling. California Agricultural Experimental Station Bulletin 670. Univ. California, USA.

De Lima, JL \& Torfs, PJ. (1994). Effects of wind on simulated rainfall and overland flow under single fullcone nozzle sprays. In: Trakiris, G., Santos, M.A. (Eds.). Proceedings of the Second European Conference on Advances in Water Resources Technology and Management. Balkema, Lisbon, Portugal. pp: 443-450.

Dechmi, F, Playán, E, Cavero, J, Faci, JM \& Martínez-Cob, A. (2003). Wind Effects on SolidSet Sprinkler Irrigation Depth and Yield of Maize (Zea mays). Irrig Sci 22(2): 67-77.

ISO Standard 15886-3. (2012). Agricultural Irrigation Equipment. Sprinklers. Part 3. Characterization of distribution and test methods. Geneva, Switzerland.

Keller, J \& Bliesner, RD. (1990). Sprinkle and Trickle Irrigation. AVI Book. Van Nostrand Reinhold. New York. USA.

$\mathrm{Li}, \mathrm{J}$ (1997). Effect of pressure and nozzle shape on the characteristics of sprinkler droplet spectra. J Agric Eng Res 66: 15-21.

Li, J, Kawano, H \& Yu, K. (1994). Droplet size distributions from different shaped sprinkler nozzles. T ASAE 37(6):1871-1878.

Montero, J, Carrión, P, Tarjuelo, JM \& Nin, R. (2006). Calibración de un Disdrómetro óptico para la medida de los tamaños de gota producidas por los aspersores. XXIV Congreso Nacional de Riego, Lugo, Spain. pp: 148-149.

Montero, J, Martínez, RS \& Tarjuelo, JM. (2004). Analysis of water application cost with permanent set sprinkler irrigation systems. Irrig Sci 23(3): 103-110.

Montero, J, Tarjuelo, JM \& Carrión, P. (2003). Sprinkler droplet size distribution measured with an optical spectropluviometer. Irrig Sci 22: 47-56.

Phocaides, A. (2007). Handbook on pressurized irrigation techniques. Food and Agriculture Organization of the United Nations (FAO). 2nd Edition. Rome, Italy.

Report (2014). Pressurized irrigation systems implemented in IRAN. Department of Development of New Irrigation Systems. Ministry of Agriculture Jihad. Iran.

Sánchez, I, Zapata, N \& Faci, JM. (2010). Combined effect of technical, meteorological and agronomical factors on solid-set sprinkler irrigation. II. Modifications of the wind velocity and of the water interception plane by the crop canopy. Agric Water Manage 97(10): 1591-1601.

Seginer, I, Nir, D \& Von Bernuth, RD. (1991a). Simulation of wind-distorted sprinkler patterns. J Irrig Drain Eng 117(2): 285- 306.

Seginer, I, Kantz, D \& Nir, D. (1991b). The distortion by wind of the distribution patterns single sprinklers. Agric Water Manage 19: 341-359.

Sheikhesmaeili, O. (2003). Analysis of the Uniformity, Evaporation and Drift Losses in SemiPortable Sprinkling Irrigation System. MSc Thesis. Univ. Shahid Chamran of Ahvaz. Iran.

Solomon, KH. (1983). Irrigation uniformity and yield theory. Doctoral Thesis. Univ. Utah State. USA.

Tarjuelo, JM, Montero, J, Carrión, PA, Honrubia, FT \& Calvo, MA. (1999a). Irrigation uniformity with medium size sprinklers Part II: influence of wind and other factors on water distribution. T ASAE 42(3): 677-689.

Tarjuelo, JM, Montero, J, Honrubia, FT, Ortiz, J \& Ortega, JF. (1999b). Analysis of uniformity of sprinkler irrigation in semi-arid area. Agric Water Manage 40(2): 315-331.

Tarjuelo, JM, Ortega, JF, Montero, J \& de Juan, JA. (2000). Modelling evaporation and drift losses in irrigation with medium size impact sprinklers under semi-arid conditions. Agric Water Manage 43: 263-284.

Yacoubi, S, Zayani, K, Slatni, A \& Playán, E. (2012). Assessing sprinkler irrigation performance using field evaluations at the Medjerda lower valley of Tunisia. Engineering 4 (10), 682-691. 